\title{
Intricate SUMO-based control of the homologous recombination machinery
}

\author{
Nalini Dhingra and Xiaolan Zhao \\ Molecular Biology Department, Memorial Sloan Kettering Cancer Center, New York, New York 10065, USA
}

The homologous recombination (HR) machinery plays multiple roles in genome maintenance. Best studied in the context of DNA double-stranded break (DSB) repair, recombination enzymes can cleave, pair, and unwind DNA molecules, and collaborate with regulatory proteins to execute multiple DNA processing steps before generating specific repair products. HR proteins also help to cope with problems arising from DNA replication, modulating impaired replication forks or filling DNA gaps. Given these important roles, it is not surprising that each HR step is subject to complex regulation to adjust repair efficiency and outcomes as well as to limit toxic intermediates. Recent studies have revealed intricate regulation of all steps of HR by the protein modifier SUMO, which has been increasingly recognized for its broad influence in nuclear functions. This review aims to connect established roles of SUMO with its newly identified effects on recombinational repair and stimulate further thought on many unanswered questions.

Homologous recombination is critical for several aspects of life, ranging from DNA repair and genome duplication to gamete production. Our understanding of HR pathways has benefited from a combination of assay systems. In cells, the generation of a defined DSB allows quantitative assessment of the status of the broken DNA molecules and the repair proteins at a temporal resolution, as well as determination of the genetic requirement for each step of repair (Haber 2016). Extensive biochemical analyses and more recently single molecule experiments have further defined the activities of HR enzymes and elucidated how they can collaborate in multiple HR steps. Several recent reviews have discussed these findings in detail (Symington et al. 2014; Heyer 2015; Ranjha et al. 2018); thus, we give only a brief overview here for each HR step to provide the context of SUMO-based regulation. As the HR machinery and its sumoylation are best examined in budding yeast, we use this system as an index for summarizing SUMO-based control. We also discuss additional

[Keywords: double-strand break repair; genome maintenance;

homologous recombination; sumoylation]

Corresponding author: zhaox1@mskcc.org

Article is online at http://www.genesdev.org/cgi/doi/10.1101/gad.328534. 119. regulation in mammalian cells and highlight their similarities and differences with those found in yeast. It is noteworthy that SUMO plays important roles in modulating protein recruitment to damaged chromatin and in other DNA break repair pathways. As these topics have been well covered in other reviews (Schwertman et al. 2016; Garvin and Morris 2017), they are not addressed here in order to maintain the focus on the regulation of core HR machinery.

Overview of the SUMO pathway and the effects of sumoylation

SUMO, a small protein of $\sim 100$ residues, is highly conserved among eukaryotes with several isoforms found in mammalian cells. SUMO can be conjugated to the lysine residues of target proteins via the action of SUMO E1, E2, and E3 enzymes (Fig. 1; Johnson 2004). The SUMO E1 helps to covalently link SUMO to the E2 enzyme's active site. In many cases, SUMO E2 can directly bind to the sumoylation consensus sequence $\psi$-K-X-E/D / $\psi$ : hydrophobic residue) or its reverse sequence on the target proteins, permitting the transfer of SUMO to the lysine residue (Rodriguez et al. 2001; Sampson et al. 2001). However, in vivo sumoylation often requires SUMO E3s, which promote productive configurations for SUMO transfer by simultaneously binding the SUMO-charged E2 and the substrate (Werner et al. 2012; Streich and Lima 2016). Organisms examined so far contain a single SUMO E1 and E2 but multiple SUMO E3s to confer some levels of substrate specificity. However, substrate overlap is also seen for SUMO E3s. Such redundancy can increase the probability of sumoylation and thus the robustness of SUMO-based regulation. Earlier genetic studies have implicated sumoylation enzymes in the regulation of $\mathrm{HR}$ and genome stability from yeast to humans (Xhemalce et al. 2004; Zhao and Blobel 2005; Branzei et al. 2006; Galanty et al. 2009; Morris et al. 2009). Further examination of how sumoylation affects substrate properties has begun to shed light on the basis of SUMO-mediated regulation.

(c) 2019 Dhingra and Zhao This article is distributed exclusively by Cold Spring Harbor Laboratory Press for the first six months after the full-issue publication date (see http://genesdev.cshlp.org/site/misc/terms.xhtml). After six months, it is available under a Creative Commons License (Attribution-NonCommercial 4.0 International), as described at http://creativecommons.org/licenses/by-nc/4.0/. 


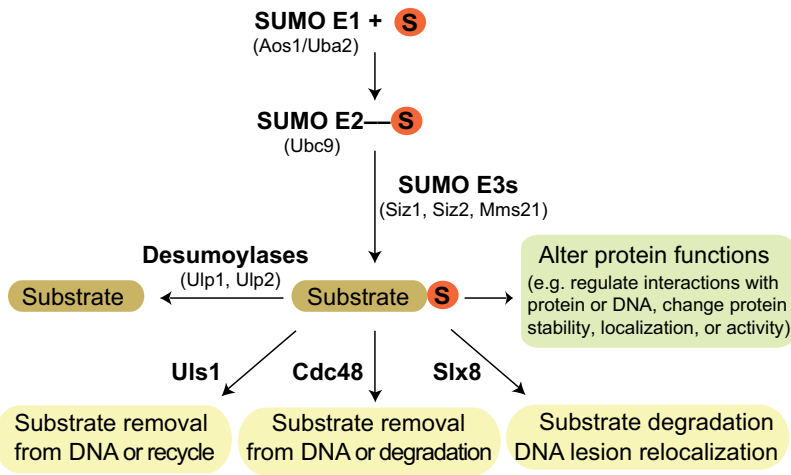

Figure 1. A simplified scheme for the SUMO pathway and consequences of sumoylation. Sumoylation of a substrate requires the sequential action of SUMO E1, E2, and E3 enzymes. The SUMO moiety (S) covalently linked to the substrates can modulate protein functions in different ways (see text for detail). Reversal of sumoylation is catalyzed by the desumoylases. The yeast SUMO pathway enzymes are indicated. In some contexts, a sumoylated substrate can be recognized by SUMO pathway accessory factors, as represented by the budding yeast proteins Uls1, $\mathrm{Cdc} 48$, and Slx5/8. These accessory proteins can regulate the fates of sumoylated proteins in distinct manners as detailed in the text.

Collective evidence suggests that the SUMO moiety can change substrate attributes in multiple ways, including altering its activities or interactions with other biomolecules (Flotho and Melchior 2013; Pichler et al. 2017). The diversity of SUMO's effects is described below in the context of each HR step. It is worthwhile to highlight a prevalent effect of sumoylation that is mediated by binding to SUMO interaction motifs (SIMs) composed of a hydrophobic core preceded or followed by negatively charged residues (Song et al. 2004; Hecker et al. 2006). The SUMO:SIM interaction is relatively weak, a feature that can actually be useful for modulating dynamic processes involving many protein hand-over events such as HR. Indeed, multiple HR factors contain SIMs, some of which have been shown to promote interactions with sumoylated proteins while others await further examination (detailed below). The SUMO:SIM interaction can also aid more stable association if additional binding interfaces are present. In some instances, stronger interactions are established when several SIMs from a protein bind to multiple SUMO moieties conjugated to the substrates or to SUMO itself (SUMO chains).

A family of multi-SIM containing proteins, STUbLs (SUMO targeted ubiquitin ligases), have a wide-ranging influence on HR by ubiquitinating sumoylated proteins or targeting the DNA bound by these proteins, such as unrepairable DNA breaks, damaged replication forks, or broken heterochromatin, to the nuclear periphery (Nie and Boddy 2016; Seeber and Gasser 2017). Another family of multi-SIM proteins, represented by the budding yeast Uls1 and fission yeast Rrp1/2, possess a SWI2/SNF2 ATPase domain and may be able to remove sumoylated proteins from chromatin via their potential translocase activities (Shah et al. 2010; Lescasse et al. 2013; Wei et al. 2017). Yet another way to extract sumoylated pro- teins from chromatin or from protein association is mediated by the Cdc48 segregase (Torrecilla et al. 2017). In both budding and fission yeasts, the Cdc48 cofactor Ufd1 uses its SIM sequences to recognize sumoylated proteins, targeting the segregase to specific substrates for their extraction and sometimes degradation (Bergink et al. 2013; Kohler et al. 2013). Collectively, STUbLs, Uls1 family proteins, and the Cdc48 segregase provide multiple avenues to regulate the fates of the SUMO targets (Fig. 1).

Competing with these accessary factors are the SUMOspecific proteases or desumoylases that can cleave SUMO off these proteins or collapse SUMO chains (Fig. 1; Hickey et al. 2012). The distinct cellular localization of these desumoylases provides an important strategy to diversify their substrate repertoire. The multiple means to remove SUMO from proteins or degrade sumoylated proteins attest to the highly dynamic nature of cellular sumoylation, and the difficulty of detecting the low levels of sumoylated forms. In addition, the so-called "on-site" sumoylation, wherein DNA metabolism factors are only sumoylated once they have engaged with DNA for specific transactions, can also explain the low levels of sumoylated forms detected in vivo (Sarangi and Zhao 2015). It is important to comprehend how the SUMO forms of substrates can exert specific effects before their elimination by the factors described above. Although multiple challenging issues remain to be addressed, studies of the sumoylation of HR proteins have provided insights into this question as summarized below.

\section{DNA end resection factors, their roles in sumoylation, and their regulation by SUMO}

The canonical HR pathway that repairs DSBs can be roughly divided into three stages that are conserved from yeast to humans. In the first stage, the DNA end resection machinery generates $3^{\prime}$ single strand DNA (ssDNA) to be used for homology searching (Symington 2016). In budding yeast, DNA end resection involves the initial trimming of 5' DNA ends by the MRX (Mre11-Rad50-Xrs2) nuclease in collaboration with Sae2, which is followed by extended DNA degradation by either the helicase-nuclease duo Sgs1 (with cofactor Top3-Rmi1) and Dna2, or the exonuclease Exo1 (Fig. 2; Symington 2016).

The resultant ssDNA is bound by the ssDNA-binding complex RPA (Rfa1-3) to prevent secondary structure formation or DNA degradation. In addition, the RPA-ssDNA filament provides a platform for recruiting the yeast SUMO E3 Siz2 that contributes to the sumoylation of RPA itself as well as the downstream HR proteins Rad52 and 59 (more below) (Chung and Zhao 2015). This recruitment requires the protein-binding module of the Rfa2 subunit and a region extended from the SAP domain of Siz2 (Chung and Zhao 2015). Since the RPA-ssDNA filament is a universal intermediate in DNA metabolic processes, it is tempting to speculate that the RPA-Siz2 interaction can also promote sumoylation in other DNA repair contexts and activate on-site sumoylation in other ssDNA 


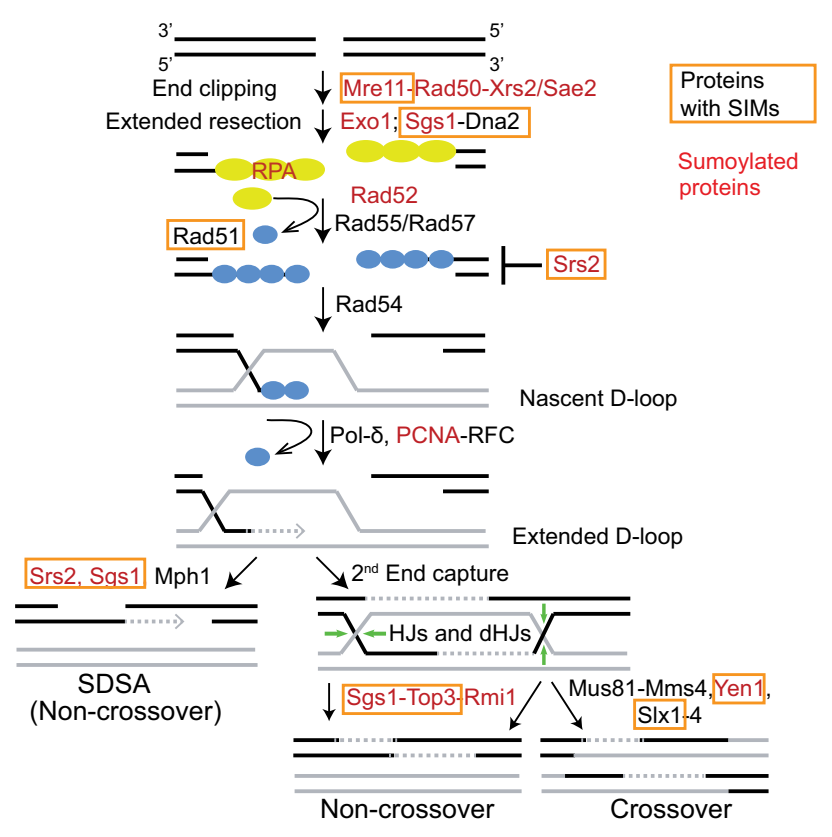

Figure 2. Summary of HR processes and HR proteins reported for sumoylation and interacting with SUMO. The figure depicts an overview of recombinational repair of DSBs with only the budding yeast proteins shown for simplicity. Recombinational repair is initiated by a two-step $5^{\prime}$ end resection process to generate $3^{\prime}$ end ssDNA, which can be coated by RPA. Several HR mediator proteins can then help to replace RPA with the Rad51 recombinase. With the assistance of Rad54, the Rad51-ssDNA filament can pair and invade donor DNA, leading to the formation of nascent D-loop structures. New DNA synthesis by Pol $\delta$ with the help of its cofactor PCNA can extend the D-loop structures, which can be subsequently processed in two different ways. In the SDSA pathway, DNA helicases, such as Srs2, Sgs1, and $\mathrm{Mph} 1$, can remove ssDNA from donor DNA, leading to noncrossover outcomes. Alternatively, second end capture can lead to the formation of Holliday junction (HJ) structures. These structures can be processed by the dissolution enzymes (Sgs1-Top3-Rmi1) to generate exclusively noncrossover products or by the resolution nucleases (Mus81-Mms4, Yen1, and Slx1/4) to produce both noncrossover and crossover products. Note that the Srs2 helicase plays multiple roles during DSB repair, and only its role in disfavoring D-loop formation is depicted here for simplicity (see the text for details). Proteins reported to be sumoylated are in red and those reported to contain SIMs are boxed in orange.

regions. As SUMO enzymes are recruited to DSB sites in mammalian cells as well (Galanty et al. 2009; Morris et al. 2009), it will be interesting to define their recruitment mechanisms and the roles of RPA in this process.

The MRX complex is also required for Siz2-mediated sumoylation of RPA and Rad52 and Rad59 (Cremona et al. 2012; Psakhye and Jentsch 2012). As lack of Sae2 and Exol has similar effects on sumoylation as mutants of MRX, these end resection factors likely contribute to sumoylation via ssDNA generation that permits Siz2 recruitment (Cremona et al. 2012; Psakhye and Jentsch 2012; Chung and Zhao 2015). This theory does not exclude another proposal that MRX may have a direct role in recruiting the SUMO machinery, which is based on yeast two-hybrid interactions of Mrel1 with SUMO, SUMO E2, and Siz2 (Chen et al. 2016). Clarifying how Mre11 associates with these proteins and its direct involvement in their recruitment will provide further insight into the roles of MRX in sumoylation. Regardless, it is noteworthy that the requirement of RPA and MRX in sumoylation reminisces their roles in DNA damage checkpoint activation. However, DNA damage-induced sumoylation and checkpoint activation are largely separable, albeit with some degree of crosstalk (Cremona et al. 2012; Wu et al. 2014). For example, eliminating the main checkpoint kinase Mec1 in yeast does not reduce bulk sumoylation, but the sumoylation of the ATRIP checkpoint protein in human cells and RPA in yeast positively affects checkpoint function (Cremona et al. 2012; Wu et al. 2014; Dhingra et al. 2019).

Not only do DNA end resection factors affect sumoylation, they are also subject to SUMO-based regulation in yeast and mammalian cells. Sumoylation has an overall positive effect on end resection, though the underlying mechanisms are only partially understood. SUMO can increase Sae2 solubility, which may be reminiscent of an "antiglue" effect of sumoylation on the polyglutaminecontaining protein ataxin-7 acting in chromatin remodeling and the aggregation-prone $\alpha$-synuclein implicated in Parkinson's disease (Janer et al. 2010; Krumova et al. 2011; Sarangi et al. 2015). SUMO exerts a stronger functional influence on the Sae2 human homolog, CtIP, by yet to be identified mechanisms (Soria-Bretones et al. 2017). In addition, sumoylation is suggested to promote the EXO1 protein stability in mammalian cells via direct or indirect means (Bologna et al. 2015). The roles of sumoylation of the yeast MRX or its mammalian counterpart MRN (MRE11-RAD50-NBS1) are also unclear, but enhancement of MRN sumoylation by an adenovirus SUMO E3 suggests a possible effect on the host-virus interaction (Cremona et al. 2012; Psakhye and Jentsch 2012; Sohn and Hearing 2012). Further studies on how SUMO influences the activities of these nucleases can also shed light on end resection in other cellular contexts, such as at stalled or collapsed replication forks, where DNA degradation needs to be tightly regulated.

\section{SUMO exerts complex control of Rad51 filament formation and functions}

The second stage of HR repair involves homology search and DNA synthesis to generate recombination intermediates (RIs) in the form of joint DNA molecules (Symington et al. 2014; Heyer 2015; Ranjha et al. 2018). First, the Rad51 recombinase replaces RPA on ssDNA, forming a presynaptic filament capable of homology search and pairing with donor DNA. Successful pairing leads to the formation of a nascent D-loop structure, which can be extended by DNA polymerase delta, resulting in the formation of an extended D-loop structure (Fig. 2). Loading Rad51 onto ssDNA requires mediator proteins, while generating D-loop structures (D-loops) depends on Rad54 and its homologs. In yeast, the most essential mediator is Rad52, with Rad55 and Rad57 also being critical in the 
repair of DSBs and the four-subunit Shu complex mostly required in replication-associated HR (Krejci et al. 2012).

Among HR proteins that catalyze the formation of D-loops, only Rad52 is known to be sumoylated (Sacher et al. 2006; Cremona et al. 2012; Psakhye and Jentsch 2012) and several models have been put forward to explain SUMO's effects on Rad52 functions. It was initially suggested that Rad52 sumoylation promotes its interaction with Rad51, favoring RI generation (Sacher et al. 2006). This notion is supported by genetic data that Rad52 sumoylation loss rescues the DNA damage sensitivity of several mutants that are sensitive to RI accumulation, such as cells lacking both Sgs1 and the Srs2 helicases that can remove Rad51 from DNA (Sacher et al. 2006). Later on, sumoylation of Rad52 was found to recruit the Cdc48 segregase to disfavor Rad52 interaction with Rad51 and promotes its degradation (Bergink et al. 2013). Other studies found that Rad52 sumoylation promotes its interaction with $\operatorname{Rad} 59$, a Rad52 paralog that catalyzes Rad51-independent repair, thus fostering Rad59-mediated repair at the expense of Rad51-mediated repair (Altmannova et al. 2010; Silva et al. 2016). Yet another model posits that sumoylation of $\operatorname{Rad} 52$ can diminish its ability to counter Srs2-mediated removal of Rad51 from ssDNA, as SUMO-fused Rad52 suppresses srs $2 \Delta$ genotoxic sensitivity without affecting overall HR repair (Esta et al. 2013). Moreover, locus-specific effects of Rad52 sumoylation have also been shown wherein it can disfavor recombination foci formation in rDNA and centromeric regions (Torres-Rosell et al. 2007; Yong-Gonzales et al. 2012). A challenge now is to formulate a unified model for the roles of Rad52 sumoylation. For example, can the pro- and antiRad51 effects mediated by Rad52 sumoylation occur at different repair or chromatin contexts, and can Cdc48-dependent extraction take place at a later stage of repair after sumoylated Rad52 carries out its mediator or Rad59-mediated functions? Addressing these questions will be critical for achieving a better understanding of how sumoylation of a single mediator protein can regulate $\mathrm{D}$-loop formation, repair pathway choices, and limit toxic recombination intermediates.

An antagonist of the HR mediator proteins, the Srs2 helicase, can both bind to SUMO and be sumoylated. Srs 2 contains a C-terminal SIM best known for collaborating with a nearby PIM (PCNA interacting motif) to bind to sumoylated PCNA (Papouli et al. 2005; Pfander et al. 2005). This interaction was initially shown to recruit Srs2 to stalled replication forks where it can remove Rad51 from DNA (Papouli et al. 2005; Pfander et al. 2005). Consequently, Srs2 can disfavor the use of canonical HR as a means to restart replication, while favoring the Rad6/18-mediated DNA tolerance pathway (Hoege et al. 2002; Stelter and Ulrich 2003). However, the story seems to be more nuanced as the Srs 2 interaction with sumoylated PCNA is not indiscriminately anti-HR but in fact favors the SDSA (synthesis dependent strand annealing) branch of recombinational repair. SDSA entails the displacement of the invaded ssDNA from extended D-loops and pairing it with the other $3^{\prime}$ ssDNA tail to allow subsequent gap synthesis and repair completion, generating ex- clusively noncrossover products (Symington et al. 2014; Heyer 2015; Ranjha et al. 2018). Alternatively, ligation of the DNA end from the D-loop with the other broken DNA end would form Holliday junction structures, the cleavage of which can lead to either crossover and noncrossover products (Fig. 2; Symington et al. 2014; Heyer 2015; Ranjha et al. 2018). As crossovers cause genetic changes of surrounding regions and lead to loss-of-heterozygosity in diploid cells, SDSA is a preferred pathway in mitotic cells.

A role for Srs2 interaction with sumoylated PCNA in SDSA is supported by both genetic and biochemical data. Mutants disrupting this interaction exhibit increased rates of crossover and loss of heterozygosity (Robert et al. 2006; Le Breton et al. 2008; Miura et al. 2013). Biochemically, while the Srs2 helicase activity can displace the invaded ssDNA from nascent or extended D-loops, sumoylated PCNA confers a bias towards the latter (Liu et al. 2017). Srs2 binding to sumoylated PCNA was also shown to prevent DNA polymerase delta binding to PCNA, disfavoring D-loop extension (Burkovics et al. 2013). These combined effects may explain the ultimate result that $\mathrm{Srs} 2$ interaction with sumoylated PCNA reduces crossover levels. An Srs2-like protein in mammals, PARI, also interacts with sumoylated PCNA, disfavors Rad51 foci formation, and HR in cells (Moldovan et al. 2012). Unlike Srs2, PARI has no classic ATPase/helicase domain and does not display helicase activity; thus, its Rad51-antagonistic effect might stem from interacting with a motor protein to displace Rad51 on ssDNA or its stimulation of the Rad51 ATPase activity that can help this disassociation (Moldovan et al. 2012).

Srs 2 is also sumoylated and eliminating this modification specifically impairs HR in the rDNA region, suggesting a unique context for Srs2 sumoylation to exert a detectable effect (Kolesar et al. 2012, 2016). Intriguingly, Srs2 sumoylation is toxic in the absence of CDK-mediated phosphorylation of Srs 2 that is thought to promote SDSA (Saponaro et al. 2010). Whether this toxicity is related to a role for Srs2 sumoylation in rDNA recombination will be interesting to explore. More recently, Srs2 sumoylation has been linked to the regulation of its protein levels. The STUbL enzyme Slx5/8 interacts with Srs 2 and can promote its degradation (Urulangodi et al. 2015). This regulation at replication forks involves another Srs2 interactor, Esc2, which associates with replication forks in vivo (Urulangodi et al. 2015). Esc2 contains two SUMO-like domains (SLD1/2) that foster its interaction with Srs2 in a manner dependent on Srs2's SIM (Urulangodi et al. 2015). The proposed model is that the two Srs2 interactors, Esc2 and Slx5/8, collaborate to reduce Srs2 levels at stalled replication forks (Urulangodi et al. 2015). This may provide a way to curb Srs2-mediated inhibition of Rad51 in situations when HR is needed to rescue replication defects. Adding to this picture, Uls1, which also interacts with $\mathrm{Slx} 5 / 8$, was recently shown to associate with Srs2 and restrain its levels as well (Kramarz et al. 2017). It will be interesting to gain a deeper understanding of the interplay between Uls1, Slx5/8, and Esc2 in Srs2 
control, such as whether they act sequentially or at distinct DNA damage sites.

Yeast and mammalian Rad51 harbor a conserved SIM, mutants of which can reduce Rad51 accretion at DNA damage sites in human cells (Bergink et al. 2013; Shima et al. 2013). It is unclear how the Rad51-SIM exerts this effect, but an obvious possibility to test is whether it binds to other sumoylated HR proteins. In human cells, the UAF1 protein containing two SLD domains can bind to a Rad51 interactor, Rad51-AP1, in a manner depending on the SIMs of Rad51-AP1 (Liang et al. 2016). This interaction fosters Rad51-mediated pairing of ssDNA with donor dsDNA in vitro and enhances HR in vivo (Liang et al. 2016). Yet another way to promote Rad51 filament formation could involve the sumoylation of human RPA (Dou et al. 2010). In summary, current studies have shown multiple layers of SUMO-based regulation of RI formation and HR pathway choices. Many questions, as exemplified above, remain to be addressed to generate unified models of how these layers of regulation can achieve specific effects in different contexts, and whether the findings in yeast are conserved in human cells.

\section{Sumoylation collaborates with phosphorylation to regulate $\mathrm{HJ}$ removal enzymes}

During the last stage of HR repair, joint DNA molecules produced during the early steps, including HJs and D-loop structures, are processed to yield linear DNA products. Multiple enzymes with RI processing activity can generate different repair outcomes and are activated at different cell cycle stages (West et al. 2015). Dissolution of double HJs (dHJs) by the STR complex or its mammalian counterpart (BLM-TopIII $\alpha-\mathrm{RMI}$ ) produces only noncrossover products; thus, it is favored in mitotic cells and is likely the main $\mathrm{HJ}$ removal activity in S phase. In addition, STR also dissolves D-loops, providing another means to remove RIs (Fasching et al. 2015; Piazza et al. 2019). In G2/M phase, the Mus81-Mms4 (human MUS81-EME1) structure-selective nuclease, which can cleave multiple types of RIs, is activated by CDK and Polo-like kinase, while the Yen1 (human GEN1) resolvase targeting single HJs is turned on only in anaphase by Cdc14-mediated dephosphorylation (Wild and Matos 2016). Both types of nucleases produce crossover and noncrossover products (Fig. 2). Thus, limiting their activities to the latter part of the cell cycle gives STR/BTR more time to generate noncrossover products, but still allows the removal of any remaining RIs before cell division. Another structure-selective DNA nuclease, the Slx1-Slx4 complex, also contributes to RI removal. The mammalian SLX4 harbors additional domains for binding to MUS81-EME1 and other proteins, gaining additional functions and regulation, which has been nicely summarized in a recent review (Guervilly and Gaillard 2018).

Recent studies have provided evidence that the aforementioned RI processing enzymes are subject to SUMObased regulation. Bulk STR sumoylation requires upstream HR factors that produce RIs, suggesting on-site sumoylation for this complex (Bonner et al. 2016; Bermu-
dez-Lopez et al. 2016). Sumoylation of STR can then engender intersubunit interaction and its accrual at DNA damage sites, thus fostering RI removal (Bonner et al. 2016; Bermudez-Lopez et al. 2016). Another study highlights the requirement of Sgs1 sumoylation at telomeres that may also mediate its role in RI removal (Lu et al. 2010). STR sumoylation partly requires the Mms21 SUMO E3 subunit of the Smc5/6 complex, explaining some shared phenotypes of Smc5/6 and STR mutants, such as RI accumulation (Bonner et al. 2016; BermudezLopez et al. 2016). Given that both Mms21 and STR also influence other processes such as replication fork regulation, it is possible that Mms21-mediated STR sumoylation may have roles beyond RI dissolution. Indeed, human BLM sumoylation, which also heavily relies on the human MMS21 homolog, appears to regulate collapsed replication forks. Sumoylation of BLM aids in its targeting to collapsed replication forks where it can both disfavor the accumulation of unproductive RIs and promote proper RAD51 functions (Ouyang et al. 2013; Pond et al. 2019). The underlying mechanisms for these two different effects remain to be elucidated.

Yen 1 is also sumoylated and this modification was suggested to foster its interaction with Uls1 (Bauer et al. 2019). Genetic data support a positive influence of Uls1 on Yen 1 function, which may explain why uls $1 \Delta$ sensitizes rad54/rdh54 mutants (Shah et al. 2010; Bauer et al. 2019). The story gets more complicated as Yen1 was also found to be ubiquitinated by Slx5/8 (Talhaoui et al. 2018). Given that both $S 1 x 5 / 8$ and Uls 1 can remove sumoylated proteins, it will be interesting to test whether they regulate Yen 1 in this manner and how such a potential effect may influence $H$ J removal. In mammalian cells, both subunits of the MUS81-EME1 nuclease have been reported to be sumoylated, and MUS81 sumoylation is suggested to prevent chromosomal misalignment in mitosis, whereas the roles of EME1 sumoylation and whether the S phase-specific EME2 subunit is sumoylated remain to be determined (Xiao et al. 2015; Hu et al. 2017).

Mammalian SLX4 contains three SIM sequences that help to target SLX4 to DNA damage sites, telomeric regions, and PML bodies (Gonzalez-Prieto et al. 2015; Guervilly et al. 2015; Ouyang et al. 2015). The SLX4 SIMs could achieve this effect by fostering interaction with multiple partner proteins known to be sumoylated, such as RPA, MRN, and the telomere protein TRF2 (Ouyang et al. 2015). Interestingly, the SIMs of SLX4 were also found to promote the sumoylation of SLX4 and one of its binding partners XPF, suggesting the possibility that SLX4 can act as a SUMO E3 or an E3 cofactor (Guervilly et al. 2015). In support of this, SLX4 specifically binds to SUMO-charged E2 but not free E2 or SUMO (Guervilly et al. 2015). As SLX4 forms megacomplexes with additional nucleases and structural proteins involved in $\mathrm{HJ}$ removal, telomere metabolism, and replication fork regulation, it will be interesting to address whether SLX 4 could broadly affect the sumoylation of its partner proteins, and whether the SIMs of SLX4 or its own sumoylation can regulate the formation of various SLX4-containing complexes and their functions. In yeast, the SUMO-binding 
function is shifted from Slx4 to Slx1 and the Slx1:SUMO interaction favors binding to the Saw1 scaffold protein involved in strand annealing pathway and the repair of UV lesions (Sarangi et al. 2014). These observations both suggest a conserved role for SUMO in the Slx1-Slx4-mediated processes and the acquisition of more sophisticated SUMO-based regulation of the human SLX4 interactome.

\section{Outlooks}

The progress in the past decade or so has revealed how extensive the involvement of SUMO is in the regulation of HR factors and pathways. Despite the difficulty of detecting low levels of sumoylated proteins, the SUMO forms of many HR factors have been demonstrated and their sumoylation mutants have been examined. These studies have suggested different effects of sumoylation in the regulation of HR protein functions as summarized above. Several HR factors also contain SIM sequences that can recruit the SUMO pathway accessory factors, such as STUbLs, the Uls1 family proteins, and the segregase complex. However, as we uncover more details about SUMObased regulation of $\mathrm{HR}$, new questions arise, some of which are summarized above. For example, how can SUMO exert multiple distinct effects on the same substrate, how do the SUMO pathway accessory factors compete with desumoylases, what determines whether a sumoylated protein affects HR functions or be extracted or removed from DNA. Answering these questions requires both cellular studies employing sumoylation defective mutants and in vitro analyses of the behaviors of sumoylated proteins and their effects on specific HR reactions. In addition, new avenues of investigation, such as more mechanistic studies in mammalian cells, and examining the possible involvement of sumoylation in forming membrane-less repair centers in the nucleus, will continue to broaden our understanding of the roles of sumoylation in genome maintenance.

\section{Acknowledgments}

We thank Prabha Sarangi for editorial comments. This work was supported by a grant from National Institute of General Medical Science (GM080670) to X.Z.

\section{References}

Altmannova V, Eckert-Boulet N, Arneric M, Kolesar P, Chaloupkova R, Damborsky J, Sung P, Zhao X, Lisby M, Krejci L. 2010. Rad52 SUMOylation affects the efficiency of the DNA repair. Nucleic Acids Res 38: 4708-4721. doi:10.1093/nar/gkq195

Bauer SL, Chen J, Astrom SU. 2019. Helicase/SUMO-targeted ubiquitin ligase Uls1 interacts with the Holliday junction resolvase Yen1. PLoS One 14: e0214102. doi:10.1371/journal .pone.0214102

Bergink S, Ammon T, Kern M, Schermelleh L, Leonhardt H, Jentsch S. 2013. Role of Cdc48/p97 as a SUMO-targeted segregase curbing Rad51-Rad52 interaction. Nat Cell Biol 15: 526532. doi:10.1038/ncb2729
Bermúdez-López M, Villoria MT, Esteras M, Jarmuz A, TorresRosell J, Clemente-Blanco A, Aragón L. 2016. Sgs1's roles in DNA end resection, $\mathrm{HJ}$ dissolution, and crossover suppression require a two-step SUMO regulation dependent on Smc5/6. Genes Dev 30: 1339-1356. doi:10.1101/gad.278275.116

Bologna S, Altmannova V, Valtorta E, Koenig C, Liberali P, Gentili C, Anrather D, Ammerer G, Pelkmans L, Krejci L, et al. 2015. Sumoylation regulates EXO1 stability and processing of DNA damage. Cell Cycle 14: 2439-2450. doi:10.1080/ 15384101.2015.1060381

Bonner JN, Choi K, Xue X, Torres NP, Szakal B, Wei L, Wan B, Arter M, Matos J, Sung P, et al. 2016. Smc5/6 mediated sumoylation of the Sgs1-Top3-Rmil complex promotes removal of recombination intermediates. Cell Rep 16: 368 378. doi:10.1016/j.celrep.2016.06.015

Branzei D, Sollier J, Liberi G, Zhao X, Maeda D, Seki M, Enomoto T, Ohta K, Foiani M. 2006. Ubc9- and Mms21-mediated sumoylation counteracts recombinogenic events at damaged replication forks. Cell 127: 509-522. doi:10.1016/j.cell.2006 .08 .050

Burkovics P, Sebesta M, Sisakova A, Plault N, Szukacsov V, Robert T, Pinter L, Marini V, Kolesar P, Haracska L, et al. 2013. Srs2 mediates PCNA-SUMO-dependent inhibition of DNA repair synthesis. EMBO I 32: 742-755. doi:10.1038/emboj .2013 .9

Chen YJ, Chuang YC, Chuang CN, Cheng YH, Chang CR, Leng $\mathrm{CH}$, Wang TF. 2016. S. cerevisiae Mre11 recruits conjugated SUMO moieties to facilitate the assembly and function of the Mre11-Rad50-Xrs2 complex. Nucleic Acids Res 44: 2199-2213. doi:10.1093/nar/gkv1523

Chung I, Zhao X. 2015. DNA break-induced sumoylation is enabled by collaboration between a SUMO ligase and the ssDNA-binding complex RPA. Genes Dev 29: 1593-1598. doi:10.1101/gad.265058.115

Cremona CA, Sarangi P, Yang Y, Hang LE, Rahman S, Zhao X. 2012. Extensive DNA damage-induced sumoylation contributes to replication and repair and acts in addition to the Mec1 checkpoint. Mol Cell 45: 422-332. doi:10.1016/j .molcel.2011.11.028

Dhingra N, Wei L, Zhao X. 2019. Replication protein A (RPA) sumoylation positively influences the DNA damage checkpoint response in yeast. J Biol Chem 294: 2690-2699. doi:10 .1074/jbc.RA118.006006

Dou H, Huang C, Singh M, Carpenter PB, Yeh ET. 2010. Regulation of DNA repair through deSUMOylation and SUMOylation of replication protein A complex. Mol Cell 39: 333-345. doi:10.1016/j.molcel.2010.07.021

Esta A, Ma E, Dupaigne P, Maloisel L, Guerois R, Le Cam E, Veaute X, Coic E. 2013. Rad52 sumoylation prevents the toxicity of unproductive Rad51 filaments independently of the anti-recombinase Srs2. PLoS Genet 9: e1003833. doi:10 .1371 /journal.pgen.1003833

Fasching CL, Cejka P, Kowalczykowski SC, Heyer WD. 2015. Top3-Rmil dissolve Rad51-mediated D loops by a topoisomerase-based mechanism. Mol Cell 57: 595-606. doi:10.1016/j .molcel.2015.01.022

Flotho A, Melchior F. 2013. Sumoylation: a regulatory protein modification in health and disease. Annu Rev Biochem 82: 357-385. doi:10.1146/annurev-biochem-061909-093311

Galanty Y, Belotserkovskaya R, Coates J, Polo S, Miller KM, Jackson SP. 2009. Mammalian SUMO E3-ligases PIAS1 and PIAS4 promote responses to DNA double-strand breaks. Nature 462: 935-939. doi:10.1038/nature08657 
Garvin AJ, Morris JR. 2017. SUMO, a small, but powerful, regulator of double-strand break repair. Philos Trans $R$ Soc Lond $B$ Biol Sci 372: 20160281. doi:10.1098/rstb.2016.0281

Gonzalez-Prieto R, Cuijpers SAG, Luijsterburg MS, van Attikum H, Vertegaal ACO. 2015. SUMOylation and PARylation cooperate to recruit and stabilize SLX4 at DNA damage sites. EMBO Rep 16: 512-519. doi:10.15252/embr.201440017

Guervilly J-H, Gaillard PH. 2018. SLX4: multitasking to maintain genome stability. Crit Rev Biochem Mol Biol 53: 475-514. doi:10.1080/10409238.2018.1488803

Guervilly JH, Takedachi A, Naim V, Scaglione S, Chawhan C, Lovera Y, Despras E, Kuraoka I, Kannouche P, Rosselli F, et al. 2015. The SLX4 complex is a SUMO E3 ligase that impacts on replication stress outcome and genome stability. Mol Cell 57: 123-137. doi:10.1016/j.molcel.2014.11.014

Haber JE. 2016. A life investigating pathways that repair broken chromosomes. Annu Rev Genet 50: 1-28. doi:10.1146/ annurev-genet-120215-035043

Hecker CM, Rabiller M, Haglund K, Bayer P, Dikic I. 2006. Specification of SUMO1- and SUMO2-interacting motifs. I Biol Chem 281: 16117-16127. doi:10.1074/jbc.M512757200

Heyer WD. 2015. Regulation of recombination and genomic maintenance. Cold Spring Harb Perspect Biol 7: a016501. doi:10.1101/cshperspect.a016501

Hickey CM, Wilson NR, Hochstrasser M. 2012. Function and regulation of SUMO proteases. Nat Rev Mol Cell Biol 13: 755766. doi:10.1038/nrm3478

Hoege C, Pfander B, Moldovan GL, Pyrowolakis G, Jentsch S. 2002. RAD6-dependent DNA repair is linked to modification of PCNA by ubiquitin and SUMO. Nature 419: 135-141. doi:10.1038/nature00991

Hu L, Yang F, Lu L, Dai W. 2017. Arsenic-induced sumoylation of Mus81 is involved in regulating genomic stability. Cell cycle 16: 802-811. doi:10.1080/15384101.2017.1302628

Janer A, Werner A, Takahashi-Fujigasaki J, Daret A, Fujigasaki H, Takada K, Duyckaerts C, Brice A, Dejean A, Sittler A. 2010. SUMOylation attenuates the aggregation propensity and cellular toxicity of the polyglutamine expanded ataxin-7. Hum Mol Genet 19: 181-195. doi:10.1093/hmg/ddp478

Johnson ES. 2004. Protein modification by SUMO. Annu Rev Biochem 73: 355-382. doi:10.1146/annurev.biochem.73.011303 .074118

Kohler JB, Jorgensen MLM, Beinoraite G, Thorsen M, Thon G. 2013. Concerted action of the ubiquitin-fusion degradation protein 1 (Ufd1) and Sumo-targeted ubiquitin ligases (STUbLs) in the DNA-damage response. PLoS One 8: e80442. doi:10 .1371/journal.pone.0080442

Kolesar P, Sarangi P, Altmannova V, Zhao X, Krejci L. 2012. Dual roles of the SUMO-interacting motif in the regulation of Srs2 sumoylation. Nucleic Acids Res 40: 7831-7843. doi:10.1093/ nar/gks484

Kolesar P, Altmannova V, Silva S, Lisby M, Krejci L. 2016. Pro-recombination role of Srs2 protein requires SUMO (small ubiquitin-like modifier) but is independent of PCNA (proliferating cell nuclear antigen) interaction. J Biol Chem 291: 7594-7607. doi:10.1074/jbc.M115.685891

Kramarz K, Mucha S, Litwin I, Barg-Wojas A, Wysocki R, Dziadkowiec D. 2017. DNA damage tolerance pathway choice through Uls1 modulation of Srs2 SUMOylation in Saccharomyces cerevisiae. Genetics 206: 513-525. doi:10.1534/genet ics.116.196568

Krejci L, Altmannova V, Spirek M, Zhao X. 2012. Homologous recombination and its regulation. Nucleic Acids Res 40: 57955818. doi:10.1093/nar/gks270
Krumova P, Meulmeester E, Garrido M, Tirard M, Hsiao H-H, Bossis G, Urlaub H, Zweckstetter M, Kugler S, Melchior F, et al. 2011. Sumoylation inhibits $\alpha$-synuclein aggregation and toxicity. I Cell Biol 194: 49-60. doi:10.1083/jcb .201010117

Le Breton C, Dupaigne P, Robert T, Le Cam E, Gangloff S, Fabre F, Veaute X. 2008. Srs2 removes deadly recombination intermediates independently of its interaction with SUMO-modified PCNA. Nucleic Acids Res 36: 4964-4974. doi:10.1093/nar/ gkn441

Lescasse R, Pobiega S, Callebaut I, Marcand S. 2013. End-joining inhibition at telomeres requires the translocase and polySUMO-dependent ubiquitin ligase Uls1. EMBO I 32: 805815. doi:10.1038/emboj.2013.24

Liang F, Longerich S, Miller AS, Tang C, Buzovetsky O, Xiong Y, Maranon DG, Wiese C, Kupfer GM, Sung P. 2016. Promotion of RAD51-mediated homologous DNA pairing by the RAD51AP1-UAF1 complex. Cell Rep 15: 2118-2126. doi:10 .1016/j.celrep.2016.05.007

Liu J, Ede C, Wright WD, Gore SK, Jenkins SS, Freudenthal BD, Todd Washington M, Veaute X, Heyer WD. 2017. Srs2 promotes synthesis-dependent strand annealing by disrupting DNA polymerase $\delta$-extending D-loops. Elife 6: e22195.

Lu C-Y, Tsai C-H, Brill SI, Teng S-C. 2010. Sumoylation of the BLM ortholog, Sgs 1, promotes telomere-telomere recombination in budding yeast. Nucleic Acids Res 38: 488-498. doi:10 $.1093 /$ nar/gkp1008

Miura T, Shibata T, Kusano K. 2013. Putative antirecombinase Srs2 DNA helicase promotes noncrossover homologous recombination avoiding loss of heterozygosity. Proc Natl Acad Sci 110: 16067-16072. doi:10.1073/pnas.1303111110

Moldovan G-L, Dejsuphong D, Petalcorin MIR, Hofmann K, Takeda S, Boulton SJ, D'Andrea AD. 2012. Inhibition of homologous recombination by the PCNA-interacting protein PARI. Mol Cell 45: 75-86. doi:10.1016/j.molcel.2011.11.010

Morris JR, Boutell C, Keppler M, Densham R, Weekes D, Alamshah A, Butler L, Galanty Y, Pangon L, Kiuchi T, et al. 2009. The SUMO modification pathway is involved in the BRCA1 response to genotoxic stress. Nature 462: 886-890. doi:10 .1038 /nature08593

Nie M, Boddy MN. 2016. Cooperativity of the SUMO and Ubiquitin pathways in genome stability. Biomolecules 6: 14 . doi:10.3390/biom6010014

Ouyang KJ, Yagle MK, Matunis MJ, Ellis NA. 2013. BLM SUMOylation regulates ssDNA accumulation at stalled replication forks. Front Genet 4: 167. doi:10.3389/fgene.2013 .00167

Ouyang J, Garner E, Hallet A, Nguyen HD, Rickman KA, Gill G, Smogorzewska A, Zou L. 2015. Noncovalent interactions with SUMO and ubiquitin orchestrate distinct functions of the SLX4 complex in genome maintenance. Mol Cell 57: 108-122. doi:10.1016/j.molcel.2014.11.015

Papouli E, Chen S, Davies AA, Huttner D, Krejci L, Sung P, Ulrich HD. 2005. Crosstalk between SUMO and ubiquitin on PCNA is mediated by recruitment of the helicase Srs2p. Mol Cell 19: 123-133. doi:10.1016/j.molcel.2005.06.001

Pfander B, Moldovan GL, Sacher M, Hoege C, Jentsch S. 2005. SUMO-modified PCNA recruits Srs2 to prevent recombination during S phase. Nature 436: 428-433. doi:10.1038/ nature 03665

Piazza A, Shah SS, Wright WD, Gore SK, Koszul R, Heyer WD. 2019. Dynamic processing of displacement loops during recombinational DNA repair. Mol Cell 73: 1255-1266. doi:10 $.1016 /$ j.molcel.2019.01.005 
Pichler A, Fatouros C, Lee H, Eisenhardt N. 2017. SUMO conjugation-a mechanistic view. Biomol Concepts 8: 13-36. doi:10.1515/bmc-2016-0030

Pond KW, de Renty C, Yagle MK, Ellis NA. 2019. Rescue of collapsed replication forks is dependent on NSMCE2 to prevent mitotic DNA damage. PLoS Genet 15: e1007942. doi:10 .1371/journal.pgen.1007942

Psakhye I, Jentsch S. 2012. Protein group modification and synergy in the SUMO pathway as exemplified in DNA repair. Cell 151: 807-820. doi:10.1016/j.cell.2012.10.021

Ranjha L, Howard SM, Cejka P. 2018. Main steps in DNA doublestrand break repair: an introduction to homologous recombination and related processes. Chromosoma 127: 187-214. doi:10.1007/s00412-017-0658-1

Robert T, Dervins D, Fabre F, Gangloff S. 2006. Mrc1 and Srs2 are major actors in the regulation of spontaneous crossover. EMBO I 25: 2837-2846. doi:10.1038/sj.emboj.7601158

Rodriguez MS, Dargemont C, Hay RT. 2001. SUMO-1 conjugation in vivo requires both a consensus modification motif and nuclear targeting. I Biol Chem 276: 12654-12659. doi:10 .1074/jbc.M009476200

Sacher M, Pfander B, Hoege C, Jentsch S. 2006. Control of Rad52 recombination activity by double-strand break-induced SUMO modification. Nat Cell Biol 8: 1284-1290. doi:10 $.1038 / \mathrm{ncb} 1488$

Sampson DA, Wang M, Matunis MJ. 2001. The small ubiquitinlike modifier-1 (SUMO-1) consensus sequence mediates Ubc9 binding and is essential for SUMO-1 modification. I Biol Chem 276: 21664-21669. doi:10.1074/jbc.M100006200

Saponaro M, Callahan D, Zheng X, Krejci L, Haber JE, Klein HL, Liberi G. 2010. Cdk1 targets Srs2 to complete synthesis-dependent strand annealing and to promote recombinational repair. PLoS Genet 6: e1000858. doi:10.1371/journal.pgen .1000858

Sarangi P, Zhao X. 2015. SUMO-mediated regulation of DNA damage repair and responses. Trends Biochem Sci 40: 233242. doi:10.1016/j.tibs.2015.02.006

Sarangi P, Altmannova V, Holland C, Bartosova Z, Hao F, Anrather D, Ammerer G, Lee SE, Krejci L, Zhao X. 2014. A versatile scaffold contributes to damage survival via sumoylation and nuclease interactions. Cell Rep 9: 143-152. doi:10.1016/j .celrep.2014.08.054

Sarangi P, Steinacher R, Altmannova V, Fu Q, Paull TT, Krejci L, Whitby MC, Zhao X. 2015. Sumoylation influences DNA break repair partly by increasing the solubility of a conserved end resection protein. PLoS Genet 11: e1004899. doi:10.1371/ journal.pgen.1004899

Schwertman P, Bekker-Jensen S, Mailand N. 2016. Regulation of DNA double-strand break repair by ubiquitin and ubiquitinlike modifiers. Nat Rev Mol Cell Biol 17: 379-394. doi:10 $.1038 / \mathrm{nrm} .2016 .58$

Seeber A, Gasser SM. 2017. Chromatin organization and dynamics in double-strand break repair. Curr Opin Genet Dev 43: 916. doi:10.1016/j.gde.2016.10.005

Shah PP, Zheng X, Epshtein A, Carey JN, Bishop DK, Klein HL. 2010. Swi2/Snf2-related translocases prevent accumulation of toxic Rad51 complexes during mitotic growth. Mol Cell 39: 862-872. doi:10.1016/j.molcel.2010.08.028

Shima H, Suzuki H, Sun J, Kono K, Shi L, Kinomura A, Horikoshi Y, Ikura T, Ikura M, Kanaar R, et al. 2013. Activation of the SUMO modification system is required for the accumulation of RAD51 at sites of DNA damage. J Cell Sci 126: 5284-5292. doi:10.1242/jcs.133744

Silva S, Altmannova V, Eckert-Boulet N, Kolesar P, Gallina I, Hang L, Chung I, Arneric M, Zhao X, Buron LD, et al. 2016.
SUMOylation of Rad52-Rad59 synergistically change the outcome of mitotic recombination. DNA Repair 42: 11-25. doi: 10 .1016/j.dnarep.2016.04.001

Sohn S-Y, Hearing P. 2012. Adenovirus regulates sumoylation of Mre11-Rad50-Nbs1 components through a paralogspecific mechanism. I Virol 86: 9656-9665. doi:10.1128/JVI $.01273-12$

Song J, Durrin LK, Wilkinson TA, Krontiris TG, Chen Y. 2004. Identification of a SUMO- binding motif that recognizes SUMO-modified proteins. Proc Natl Acad Sci 101: 1437314378. doi:10.1073/pnas.0403498101

Soria-Bretones I, Cepeda-Garcia C, Checa-Rodriguez C, Heyer V, Reina-San-Martin B, Soutoglou E, Huertas P. 2017. DNA end resection requires constitutive sumoylation of CtIP by CBX4. Nat Commun 8: 113. doi:10.1038/s41467-017-00183-6

Stelter P, Ulrich HD. 2003. Control of spontaneous and damageinduced mutagenesis by SUMO and ubiquitin conjugation. Nature 425: 188-191. doi:10.1038/nature01965

Streich FC Jr, Lima CD. 2016. Capturing a substrate in an activated RING E3/E2-SUMO complex. Nature 536: 304-308. doi:10 $.1038 /$ nature 19071

Symington LS. 2016. Mechanism and regulation of DNA end resection in eukaryotes. Crit Rev Biochem Mol Biol 51: 195212. doi:10.3109/10409238.2016.1172552

Symington LS, Rothstein R, Lisby M. 2014. Mechanisms and regulation of mitotic recombination in Saccharomyces cerevisiae. Genetics 198: 795-835. doi:10.1534/genetics.114 .166140

Talhaoui I, Bernal M, Mullen JR, Dorison H, Palancade B, Brill SJ, Mazon G. 2018. Slx5-Slx8 ubiquitin ligase targets active pools of the Yen1 nuclease to limit crossover formation. Nat Commun 9: 5016. doi:10.1038/s41467-018-07364-x

Torrecilla I, Oehler J, Ramadan K. 2017. The role of ubiquitin-dependent segregase $\mathrm{p} 97$ (VCP or Cdc48) in chromatin dynamics after DNA double strand breaks. Philos Trans $R$ Soc Lond $B$ Biol Sci 372: 20160282. doi:10.1098/rstb.2016.0282

Torres-Rosell J, Sunjevaric I, De Piccoli G, Sacher M, EckertBoulet N, Reid R, Jentsch S, Rothstein R, Aragon L, Lisby M. 2007. The Smc5-Smc6 complex and SUMO modification of Rad52 regulates recombinational repair at the ribosomal gene locus. Nat Cell Biol 9: 923-931. doi:10.1038/ncb1619

Urulangodi M, Sebesta M, Menolfi D, Szakal B, Sollier J, Sisakova A, Krejci L, Branzei D. 2015. Local regulation of the Srs2 helicase by the SUMO-like domain protein Esc2 promotes recombination at sites of stalled replication. Genes Dev 29: $2067-$ 2080. doi:10.1101/gad.265629.115

Wei Y, Diao L-X, Lu S, Wang H-T, Suo F, Dong M-Q, Du L-L. 2017. SUMO-targeted DNA translocase Rrp2 protects the genome from Top2-induced DNA damage. Mol Cell 66: 581596.e586. doi:10.1016/j.molcel.2017.04.017

Werner A, Flotho A, Melchior F. 2012. The RanBP2/RanGAP1* SUMO1/Ubc9 complex is a multisubunit SUMO E3 ligase. Mol Cell 46: 287-298. doi:10.1016/j.molcel.2012.02.017

West SC, Blanco MG, Chan YW, Matos J, Sarbajna S, Wyatt HDM. 2015. Resolution of recombination intermediates: mechanisms and regulation. Cold Spring Harb Sym Quant Biol 80: 103-109. doi:10.1101/sqb.2015.80.027649

Wild P, Matos J. 2016. Cell cycle control of DNA joint molecule resolution. Curr Opin Cell Biol 40: 74-80. doi:10.1016/j.ceb .2016 .02 .018

Wu CS, Ouyang J, Mori E, Nguyen HD, Marechal A, Hallet A, Chen DI, Zou L. 2014. SUMOylation of ATRIP potentiates DNA damage signaling by boosting multiple protein interactions in the ATR pathway. Genes Dev 28: 1472-1484. doi:10 $.1101 / \operatorname{gad} .238535 .114$ 
Xhemalce B, Seeler JS, Thon G, Dejean A, Arcangioli B. 2004. Role of the fission yeast SUMO E3 ligase Plilp in centromere and telomere maintenance. EMBO J 23: 3844-3853. doi:10 $.1038 /$ sj.emboj. 7600394

Xiao Z, Chang J-G, Hendriks IA, Sigurdsson JO, Olsen JV, Vertegaal ACO. 2015. System-wide analysis of SUMOylation dynamics in response to replication stress reveals novel small ubiquitin-like modified target proteins and acceptor lysines relevant for genome stability. Mol Cell Proteomics 14: 1419-1434. doi:10.1074/mcp.O114.044792
Yong-Gonzales V, Hang LE, Castellucci F, Branzei D, Zhao X. 2012. The Smc5-Smc6 complex regulates recombination at centromeric regions and affects kinetochore protein sumoylation during normal growth. PLoS One 7: e51540. doi:10.1371/ journal.pone.0051540

Zhao X, Blobel G. 2005. A SUMO ligase is part of a nuclear multiprotein complex that affects DNA repair and chromosomal organization. Proc Natl Acad Sci 102: 4777-4782. doi:10.1073/ pnas.0500537102 


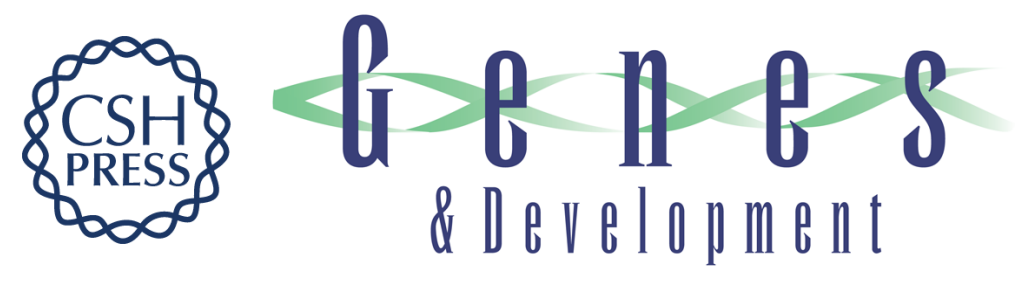

\section{Intricate SUMO-based control of the homologous recombination machinery}

Nalini Dhingra and Xiaolan Zhao

Genes Dev. 2019, 33:

Access the most recent version at doi:10.1101/gad.328534.119

\begin{tabular}{|c|c|}
\hline References & $\begin{array}{l}\text { This article cites } 86 \text { articles, } 21 \text { of which can be accessed free at: } \\
\text { http://genesdev.cshlp.org/content/33/19-20/1346.full.html\#ref-list-1 }\end{array}$ \\
\hline $\begin{array}{l}\text { Creative } \\
\text { Commons } \\
\text { License }\end{array}$ & $\begin{array}{l}\text { This article is distributed exclusively by Cold Spring Harbor Laboratory Press for the first } \\
\text { six months after the full-issue publication date (see } \\
\text { http://genesdev.cshlp.org/site/misc/terms.xhtml). After six months, it is available under a } \\
\text { Creative Commons License (Attribution-NonCommercial } 4.0 \text { International), as described } \\
\text { at http://creativecommons.org//icenses/by-nc/4.0/. }\end{array}$ \\
\hline $\begin{array}{l}\text { Email Alerting } \\
\text { Service }\end{array}$ & $\begin{array}{l}\text { Receive free email alerts when new articles cite this article - sign up in the box at the top } \\
\text { right corner of the article or click here. }\end{array}$ \\
\hline
\end{tabular}

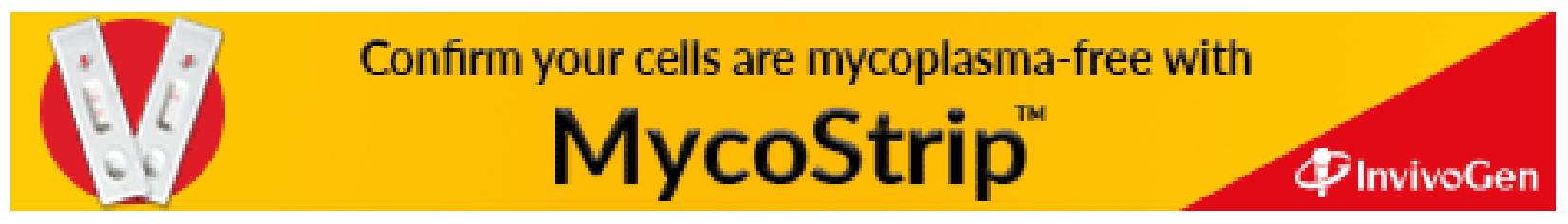

